\title{
An investigation of intravenous ethanol self-administration in rats using a fixed ratio schedule of reinforcement
}

\author{
L. A. GRUPP \\ Department of Pharmacology, University of Toronto, Toronto, Ontario M5S 1A8, Canada \\ and Neurobiology Department, Addiction Research Foundation, Toronto, Ontario M5S 2S1, Canada
}

\begin{abstract}
Six rats were trained to press one (food) bar under a 30-sec variable interval (VI) schedule for food pellets. Once responding had stabilized, a second (drug) bar was introduced and responding on it produced intravenous infusions of ethanol according to a fixed ratio (FR) schedule of reinforcement. Across a range of ethanol doses $(1-180 \mathrm{mg} / \mathrm{kg})$, increasing the FR requirement yielded an unsystematic picture of increases, decreases, and no change in response output on the drug bar. When, in two of these animals, cocaine hydrochloride (.5-1.5 mg/ kg) was tested subsequent to the ethanol, any increase in FR requirement was usually accompanied by some increase in response output. These findings suggest that the intravenous administration of ethanol is a relatively weak positive reinforcer in that it does not support self-administration behavior under conditions which otherwise do support the intravenous self-administration of cocaine.
\end{abstract}

A number of studies have reported that drug-naive monkeys and rats will self-administer ethanol by the intravenous route, sometimes to the point of gross intoxication (Deneau, Yanagita, \& Seevers, 1969; Denoble \& Begleiter, 1978; Karoly, Winger, Ikomi, \& Woods, 1978; Smith, Werner, \& Davis, 1976; Winger \& Woods, 1973; Woods, Ikomi, \& Winger, 1971). At the same time, there are reports that raise some questions about the reinforcing properties of ethanol. These center around the findings that: (1) a fairly large proportion of both rats and monkeys (one-third to one-half) fail at the outset to initiate ethanol self-administration (e.g., Altshuler, Phillips, \& Feinhandler, 1980; Smith \& Davis, 1974; Winger \& Woods, 1973); (2) monkeys will abruptly terminate self-administration, and the subsequent onset of withdrawal is not always a sufficient condition to reinstate self-administration behavior (Deneau et al., 1969); and (3) even when responding for ethanol has been initiated with the aid of behavioral techniques [e.g., schedule induced self-injection (Oei \& Singer, 1979)] it is not maintained once these behavioral manipulations are terminated. In most of the above studies, first an operant rate for infusions of the vehicle, usually saline, is established and then

The author wishes to acknowledge the excellent assistance of Ed Perlanski throughout all phases of this experiment. Thanks are also due Val Cabral, for typing the manuscript, and E. V. Wilson, Bureau of Dangerous Drugs, Ottawa, through whose offices we obtained the cocaine hydrochloride. H. Kalant and M. A. Linseman were kind enough to read the manuscript and provide useful criticism. ethanol is substituted and any subsequent changes in response rate on an FR1 schedule are monitored. If the rate shows an increase from vehicle levels, this is considered evidence for the positive reinforcing properties of the drug.

In this report, we assess the ability of intravenously administered ethanol to act as a reinforcing stimulus, using a different and complementary measure which does not use as a reference the operant levels of barpressing for the vehicle. Thus, if the intravenous administration of ethanol is, indeed, a positively reinforcing stimulus, then, providing a suitable dose is used, animals ought to adjust their response output accordingly when the response-toreinforcement (FR) requirement is changed. Such a measure has already been used to demonstrate the ability of cocaine infusions to maintain barpressing behavior on a variety of FR schedules (e.g., Pickens \& Thompson, 1968; Ross, Laska, \& Fennessy, 1978). The present study is a preliminary attempt to describe the changes in response output that occur when both ethanol dose and FR requirement are varied. Since ethanol consumption in humans often takes place concurrently with other consumatory behaviors, such as eating, and in view of the evidence indicating that ethanol self-administration in rats is facilitated by the presence of food (Meisch \& Henningfield, 1977; Oei \& Singer, 1979), we introduced a VI schedule for food operating on one bar concurrently with the FR schedule for ethanol operating on a second bar. Finally, in order to verify that these conditions were indeed sufficient to foster drug self-administration, the effect of these same 
schedule changes on responding maintained by cocaine infusions was also tested.

\section{METHOD}

\section{Subjects}

The subjects were six experimentally naive male Wistar rats weighing between 300 and $400 \mathrm{~g}$ at the beginning of the experiment. They were maintained on a 12-h-light (7 a.m.-7 p.m.), 12-h-dark cycle and housed in individual cages between sessions.

\begin{abstract}
Apparatus
A rectangular chamber, measuring $11 \frac{1 / 2}{2} \times 91 / 2 \times 10^{3 / 4}$ in., was equipped with two levers located on adjacent walls, a food cup, a Richter tube, stimulus lights, and a speaker, and was enclosed within a ventilated sound-attenuating chamber. A mercury swivel located atop the chamber was connected to a light wire cable which passed to the inner cage and could be attached to an amphenol connector on the animal's head. A drug swivel (Brown, Amit, \& Weeks, 1976) adjacent to the mercury swivel was connected at one end to an infusion pump; at the other end it was connected, by means of a polyethylene delivery tube that straddled the cable, to the animal. Such a system protected the delivery tube and permitted the infusion of drugs without interfering with the ability of the animal to respond on the bar, eat, or drink.
\end{abstract}

\section{Procedure}

Under Nembutal anesthesia $(50 \mathrm{mg} / \mathrm{kg})$, the silastic portion of a one-way silastic-polyethylene catheter was implanted in the jugular vein, with the polyethylene portion passing around the forepaw to exit at the nape of the neck. At the same time, a nine-pin amphenol microconnector with a 1-in. length of larger diameter silastic tubing attached at its rear was fixed to the skull with dental cement. The catheter was then treaded through this silastic tubing to rest at the top of the connector. Details of the catheter construction and implantation can be found elsewhere (Grupp, 1980).

Approximately 3 days after catheterization, the animals were put on restricted feeding. When they reached $85 \%$ of their freefeeding weights, they were introduced into the chamber, hooked up to the swivel device, and shaped to press a bar to obtain food on a FR1 schedule. Each reinforcement consisted of one 45-mg Noyes pellet and was accompanied by a 1-sec "lights-out" period. When this response was well established, the FR schedule was changed to a VI 30 -sec schedule and three daily 90 -min sessions followed. At this point, the (second) drug bar was introduced.

The doses of ethanol used were 1.0, 5.0, 10.0, 30.0,60.0, 120, and $180 \mathrm{mg} / \mathrm{kg}$; they were presented in a random order. Concentrations ranged from $.4 \%(\mathrm{w} / \mathrm{v})$ for the lightest animal at the lowest dose to $52 \%(\mathrm{w} / \mathrm{v})$ for the heaviest animal at the highest dose. All doses of the drug were freshly prepared in saline. The range of doses was chosen to encompass those which other investigators (Oei \& Singer, 1979; Smith, Werner, \& Davis, 1976) had found effective. Drug delivery consisted of a $3-\mathrm{sec}$ infusion of a $.1-\mathrm{ml}$ solution and was accompanied by a tone of equal duration. Responses occurring during the infusion were counted but had no programmed consequences. The drug bar always operated on a FR schedule whose requirement, presented in ascending order, ranged from one to eight responses. At any one dose of ethanol, each animal was tested for three successive sessions at a particular FR requirement, and this dose-ratio combination was repeated on three successive sessions. The FR requirement was then increased, and another set of three sessions at this dose-ratio combination was repeated. In this way, each animal was tested repeatedly on a variety of dose-ratio combinations. Both the food and drug bars, then, were completely subject to the animal's choice and operated concurrently under VI 30-sec (food)-FRI-8 (drug) schedules, respectively. Loss of cannula patency or animal death prevented the testing of all animals at all dose-ratio combinations. Water in the Richter tubes was freely available throughout the sessions, which were run 5 days a week during the light cycle and were $90 \mathrm{~min}$ in duration. Supplementary pellets of Purina Rat Chow were given in the home cages to maintain the animals' weights. For four of the six animals, three sessions of concurrent VI 30-sec-FR1 saline were run prior to the ethanol sessions.

Three doses of cocaine hydrochloride prepared in saline (.5, $1.0,1.5 \mathrm{mg} / \mathrm{kg}$ in $.1 \mathrm{ml}$ ) were subsequently substituted for ethanol in two animals who were the last to be tested with ethanol, and the gamut of dose-ratio combinations was retested. All other conditions and contingencies remained in effect during this cocaine phase.

\section{RESULTS}

\section{Effect of FR Requirement on Response Output}

Figure 1 shows the effects of varying the FR requirement on response output for each animal at all doses of ethanol and cocaine.

When ethanol was made contingent on responding (Figure 1A), the main effect of increasing ratio size was to produce a variety of increases, decreases, or no change in response output, none of which were dose specific, systematic, or consistently seen in all the animals. The doses most frequently tested were 10,30 , and $120 \mathrm{mg} / \mathrm{kg}$. For the $10-$ and $120-\mathrm{mg} / \mathrm{kg}$ doses, responding increased with increasing ratio requirement in three animals and decreased in two; for the $30-\mathrm{mg} / \mathrm{kg}$ dose, responding increased in one and decreased in four. Increasing the requirement from one to three responses in the 23 dose-ratio combinations tested in this experiment produced corresponding rate increases in only 10 of the combinations. When further testing was carried out in some of the animals at FR5, four of five rats (i.e., R33, $30 \mathrm{mg} / \mathrm{kg}$; $\mathrm{R} 24,120 \mathrm{mg} / \mathrm{kg}$; and $\mathrm{R} 34$ and $\mathrm{R} 35,60 \mathrm{mg} / \mathrm{kg}$ ) not only failed to show an increase in output to match the increase in response requirement, but, in fact, showed decreases to rates approximating those observed at FR1.

In four of the six animals, operant rates for the saline vehicle were established immediately prior to the introduction of the ethanol. These rates were almost always above the FR1 rates for many of the ethanol doses and ranged from an average of .125 to .525 responses $/ \mathrm{min}$.

Figure 1B presents the effects on FR responding when different doses of cocaine were substituted for ethanol in two of the previously tested animals (R35, $\mathrm{R} 36)$. In contrast to ethanol, increasing the ratio requirement produced an accompanying and significant increase in response output for both animals in all but one case (i.e., $1.0 \mathrm{mg} / \mathrm{kg}$ for $\mathrm{R} 35$ ) $[\mathrm{F}(1,13)$ $=5.4, p<.05]$. When both animals were tested at ratio requirements greater than those used with ethanol, that is, FR8 and FR12, response output continued to increase. In R36, good FR responding was maintained by all three doses at all the FR values 

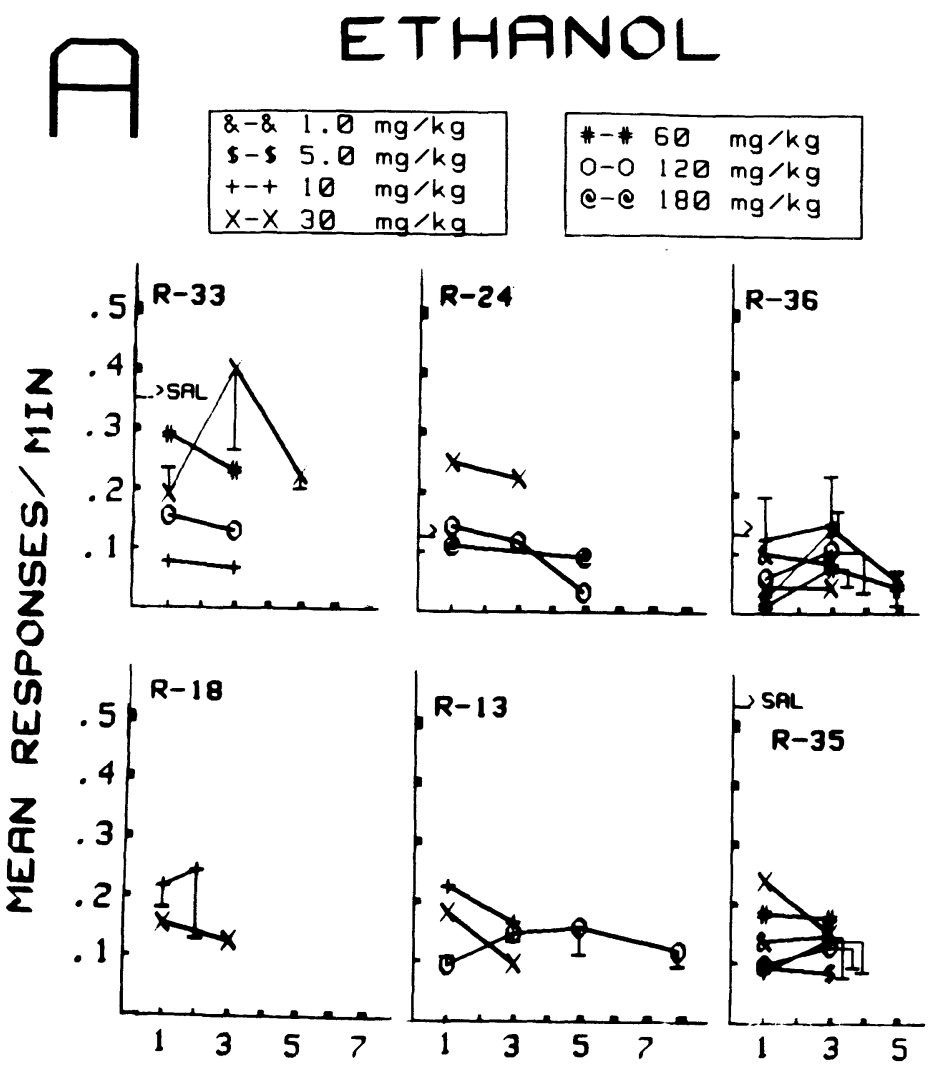

FIXED RATIO VALUE

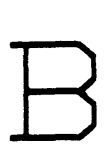

COCAINE

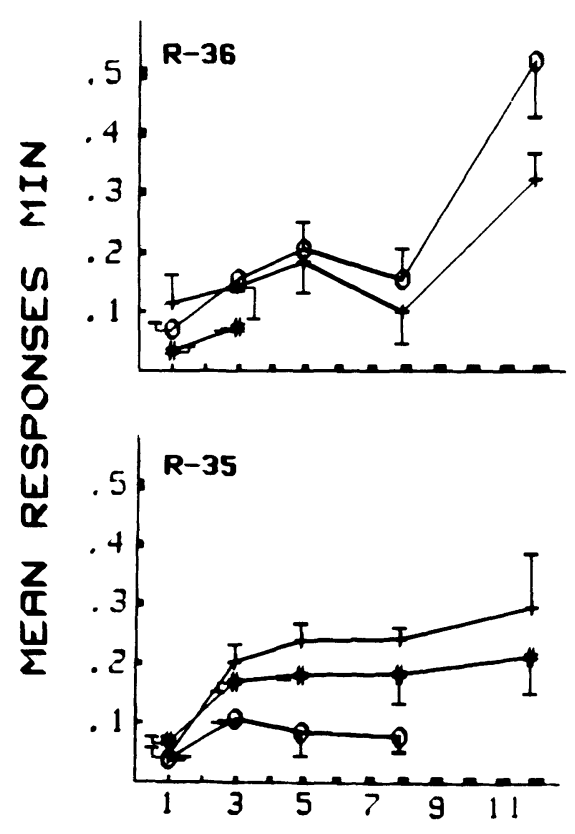

FIXED RATIO VALUE

Figure 1. Effects of fixed ratio value on response rate for various doses of ethanol (A) and cocaine (B). Each point represents the mean response rate derived from three successive sessions at that particular dose-ratio combination. Vertical lines indicate positive or negative half of the S.E.M. For clarity, these are presented only at doses at which the animals showed a tendency to increase response rate with an increase in ratio requirement. _ _ indicates the mean response rate for saline, obtained prior to the introduction of ethanol.

tested, while for $\mathrm{R} 35$, the $1.0-\mathrm{mg} / \mathrm{kg}$ dose was apparently not as potent as the other two.

\section{Effects of FR Requirements on Number of Infusions}

Table 1 gives the number of ethanol and cocaine infusions obtained for each animal at each dose, expressed as a percentage of the number of infusions obtained at FR1 for that dose.

Almost all of the animals tested with ethanol received far fewer infusions at FR3 than they did at FR1. Furthermore, no particular dose of ethanol consistently maintained a greater number of infusions than any other. Cocaine was better able to maintain FR1 levels of infusions when the requirement was shifted to FR3 than did ethanol, but again not so completely as to prevent a decrease in total drug intake. However, averaged across dose, at FR3 ethanol yielded $51.5 \% \pm 9.16$ of the FR1 infusions while cocaine yielded $87.3 \% \pm 14.81$, a difference that was statistically significant $[\mathrm{t}(24)=1.98, \mathrm{p}<.02)$. Fewer animals were tested with ethanol at FR5, but those that were obtained even fewer infusions than they did at FR3. There was also a tendency for the animals tested with cocaine to obtain fewer infusions in transition from FR3 to FR5, but this drug continued to maintain significantly greater numbers of infusions at this ratio than did ethanol $[\mathrm{t}(7)=2.52, \mathrm{p}<.01]$. Furthermore, when the ratio requirement was increased to FR8 and FR12, the number of cocaine infusions did not significantly decrease $[\mathrm{t}(3)=.503$, n.s.].

\section{Effect of the Drugs on Barpress Behavior}

The data from R35 and R36, the only two animals tested with both ethanol and cocaine, were examined in order to ascertain whether these drugs, per se, had any effect on the animals' general ability to perform the barpress response. For both rats, a regression analysis indicated no significant tendency for either overall response rate for food $[F(1,36)=.7$, n.s.; $\mathrm{F}(1,39)=.04$, n.s.] or total number of food reinforcements obtained $[F(1,36)=1.07$, n.s.; $F(1,39)=.4$, n.s.] to vary systematically with the amount (in $\mathrm{mg} / \mathrm{kg}$ ) 
Table 1

Relative Number of Infusions (Percentage of FR1 Infusions) for All Animals at Each Dose-Ratio Combination Tested

\begin{tabular}{|c|c|c|c|c|c|c|c|c|c|c|}
\hline \multicolumn{5}{|c|}{ Ethanol } & \multicolumn{6}{|c|}{ Cocaine } \\
\hline \multirow[b]{2}{*}{ Dose* } & \multirow[b]{2}{*}{ Rat } & \multicolumn{3}{|c|}{ Ratio } & \multirow[b]{2}{*}{ Dose* } & \multirow[b]{2}{*}{ Rat } & \multicolumn{4}{|c|}{ Ratio } \\
\hline & & FR3 & FR5 & FR8 & & & FR3 & FR5 & FR8 & FR12 \\
\hline 1 & $\begin{array}{l}35 \\
36\end{array}$ & $\begin{array}{l}35.3 \\
41.1\end{array}$ & & & .5 & $\begin{array}{l}35 \\
36\end{array}$ & $\begin{array}{r}141.8 \\
42.3\end{array}$ & $\begin{array}{r}100.0 \\
38.4\end{array}$ & $\begin{array}{l}66.8 \\
11.5\end{array}$ & $\begin{array}{l}41.8 \\
26.9\end{array}$ \\
\hline 5 & $\begin{array}{l}35 \\
36\end{array}$ & $\begin{array}{r}34.9 \\
166.5\end{array}$ & 33.5 & & 1.0 & $\begin{array}{l}35 \\
36\end{array}$ & $\begin{array}{r}100.0 \\
76.4\end{array}$ & $\begin{array}{l}33.3 \\
64.7\end{array}$ & $\begin{array}{l}11.0 \\
29.5\end{array}$ & 70.5 \\
\hline 10 & $\begin{array}{l}35 \\
36 \\
33 \\
13\end{array}$ & $\begin{array}{l}64.7 \\
44.1 \\
22.2 \\
23.8\end{array}$ & & & 1.5 & $\begin{array}{l}35 \\
36\end{array}$ & $\begin{array}{l}89.0 \\
74.9\end{array}$ & 57.1 & 31.8 & 28.6 \\
\hline 30 & $\begin{array}{l}35 \\
36 \\
33 \\
13 \\
18 \\
24\end{array}$ & $\begin{array}{l}24.1 \\
30.0 \\
66.6 \\
15.5 \\
30.6 \\
41.9\end{array}$ & 20.8 & & & & & & & \\
\hline 60 & $\begin{array}{l}35 \\
36 \\
33\end{array}$ & $\begin{array}{r}30.8 \\
167.0 \\
34.7\end{array}$ & & & & & & & & \\
\hline 120 & $\begin{array}{l}35 \\
36 \\
13 \\
24\end{array}$ & $\begin{array}{l}58.0 \\
58.3 \\
59.3 \\
26.1\end{array}$ & $\begin{array}{r}34.7 \\
4.3\end{array}$ & 12.7 & & & & & & \\
\hline
\end{tabular}

*In milligrams per kilogram of body weight.

of ethanol self-administered. For R35, there was a significant tendency for overall response rate for food to increase with increasing amounts of selfadministered cocaine $[\mathrm{F}(1,41)=56.6, \mathrm{p}<.01]$, although no such tendency was evident for $R 36[F(1,36)$ $=.04$, n.s.]. The number of food reinforcements obtained in both rats did not show any systematic variation with the amount of self-administered cocaine $[\mathrm{F}(1,41)=.4$, n.s.; $\mathrm{F}(1,36)=.2$, n.s. $]$.

\section{DISCUSSION}

The present study attempted to assess the ability of a number of intravenously administered doses of ethanol to act as a reinforcing stimulus using a technique requiring that the animals adjust their barpressing output according to the requirements of a FR schedule of reinforcement. We found that, under the conditions of this experiment, the intravenous administration of a wide range of ethanol doses showed little if any positive reinforcing properties in that they were weak in promoting the appropriate adjustments in response output when changes in the fixed ratio requirement were instituted. In contrast, animals responding under identical conditions for cocaine infusions adjusted their response output much more effectively to the change in response requirement and did so rapidly, indicating that the cocaine infusions were positively reinforcing.
As far as we can tell, the differences in the adjustments of response output for ethanol and cocaine are unlikely to be related to the differences in the general behavioral effects of stimulants and depressants. In most cases, both drugs had very little effect, if any, on the response rate for food or on the total number of food reinforcements obtained. Within any one dose of cocaine, the response output continued to increase with the increasing ratio requirement, although the absolute number of infusions either remained the same or decreased. This is not congruent with the notion that the psychomotor effects of cocaine were responsible for an overall increase in response rate. With respect to ethanol, there was no tendency for response rate on the food schedule or for the number of food reinforcements obtained to vary with the amount of ethanol infused. This indicates that whatever depressant (or stimulant) effects ethanol may have had, they were insufficient to either disrupt general barpressing behavior or to interfere with those processes that govern the way that behavior is patterned. Taken together, these findings indicate that neither competing behavioral effects elicited by either drug or the testing conditions themselves can account for the differences in self-administration behavior of the two drugs.

Saline determinations were obtained first and then were followed immediately by the availability of ethanol. Thus, without the opportunity for saline "re- 
sponding" to extinguish, any sudden change in response rate at this point of substitution would reflect, to some degree, the animal's initial reaction to the ethanol stimulus. The finding, in three of four animals, of an immediate drop in the FR1 response rate for ethanol is an indication that perhaps some aspect of intravenously administered ethanol is aversive (see Grupp, in press). Whether this is related to the central or peripheral effects of the drug has yet to be determined.

A number of other self-administration studies using both monkeys and rats have indicated that, while most animals may eventually be "trained," usually through drug substitution, to self-administer ethanol intravenously, only about one-third to onehalf will actually initiate ethanol self-administration when tested on a FR1 schedule (Altshuler et al., 1980; Deneau et al., 1969; Numan, 1980; Smith et al., 1976; Winger \& Woods, 1973; Woods et al., 1971). On the other hand, other investigators (Carney, Llewellyn, \& Woods, 1976; Denoble \& Begleiter, 1978) report no difficulties in training their monkeys to self-inject ethanol and indicate that the animals will show substantial response rates on such intermittent schedules as FR10 and VI 2 min, respectively. The present findings, using a different method for assessing intravenous ethanol self-administration behavior, taken together with the above studies highlight the wide divergence in the degree to which ethanol as a reinforcing stimulus can be shown to acquire and maintain control over behavior and emphasize the sensitive nature of intravenous ethanol selfadministration as a phenomenon. This is in rather sharp contrast to the self-administration of other psychoactive drugs, such as cocaine, for which drugdirected behavior is much stronger and more reliable (e.g., the present study; Goldberg, Hoffmeister, Schlitchting, \& Wuttke, 1971; Pickens \& Thompson, 1968). It is in agreement with other rat and monkey studies using other depressants, such as morphine (Weeks \& Collins, 1978) and pentobarbital (Goldberg et al., 1971), which also report poor matching of response output to the schedule requirement.

\section{REFERENCES}

Altshuler, H. L., Phillips, P. E., \& Feinhandler, D. A. Alteration of ethanol self-administration by naltrexone. Life Sciences, 1980, 26, 679-688.

Brown, Z. W., Амiт, Z., \& Weeks, J. R. Simple flow-thru swivel for infusions into unrestrained animals. Pharmacology, Biochemistry and Behavior, 1976, 5, 363-365.

Carney, J. M., Llewellyn, M. E., \& Woods, J. H. Variable interval responding maintained by intravenous codeine and ethanol injections in the rhesus monkey. Pharmacology, Biochemistry and Behavior, 1976, 5, 577-582.

Deneau, G. A., Yanagita, T., \& Seevers, M. H. Selfadministration of psychoactive substances by monkey: A measure of psychological dependence. Psychopharmacologia, 1969, 16, 30-48.

Denoble, V. J., \& Begleiter, H. Alcohol self-administration in monkeys (Macaca radiata): The effects of prior alcohol exposure. Pharmacology, Biochemistry and Behavior, 1978, 8, 391-397.

Goldberg, S. R., Hoffmeister, F., Schlitchting, U. U., \& WuTtKe, W. A comparison of pentobarbital and cocaine self-administration in rhesus monkeys: Effects of dose and fixed-ratio parameter. Journal of Pharmacology and Experimental Therapeutics, 1971, 179, 277-283.

GruPP, L. A. Biphasic action of ethanol on single units of the dorsal hippocampus and the relationship to the cortical EEG. Psychopharmacology, 1980, 70, 95-103.

GRUPP, L. A. Ethanol as the negative reinforcer in an active avoidance paradigm. Progress in Neuro-Psychopharmacology, in press.

Karoly, A. J., Winger, G., Ikomi, F., \& Woods, J. H. The reinforcing property of ethanol in the Rhesus monkey: II. Some variables related to the maintenance of intravenous ethanolreinforced responding. Psychopharmacology, 1978, 58, 19-25.

Meisch, R. A., \& Henningfield, J. E. Drinking of ethanol by Rhesus monkeys: Experimental strategies for establishing ethanol as a reinforcer. In M. M. Gross (Ed.), Alcohol intoxication and withdrawal-IIIb. New York: Plenum Press, 1977.

NumAN, R. Intravenous self-administration of ethanol maintains physical dependence in rats. Society for Neuroscience Abstracts, $1980,6,47$.

Oei, T. P. S. \& Singer, G. Effects of a fixed time schedule and body weight on ethanol self-administration. Pharmacology, Biochemistry and Behavior, 1979, 10, 767-770.

Pickens, R., \& Thompson, T. Cocaine-reinforced behaviour in rats: Effects of reinforcement magnitude and fixed-ratio size. Journal of Pharmacology and Experimental Therapeutics, 1968, 161, 122-129.

Ross, J. W., Laska, F. J., \& Fennessy, M. R. Brain biogenic amines and intravenous self-administration of cocaine in rats. Clinical and Experimental Pharmacology and Physiology, 1978, 5, 351-359.

Sмiтh, S. G., \& Davis, W. M. Intravenous alcohol selfadministration in the rat. Pharmacological Research Communications, 1974, 6, 397-401.

Smith, S. G., Werner, T. E., \& Davis, W. M. Comparison between intravenous and intragastric alcohol self-administration. Physiological Psychology, 1976, 4, 91-93.

WEEKS, J. R., \& Collins, R. J. Self-administration of morphine in the rat: Relative influence of fixed-ratio and time-out. Pharmacology, Biochemistry and Behavior, 1978, 9, 703-704.

Winger, G. D., \& Woods, J. H. The reinforcing property of ethanol in the rhesus monkey: I. Initiation, maintenance and termination of intravenous ethanol-reinforced responding. Annals of the New York Academy of Sciences, 1973, 215, 162-175.

Woods, J. H., Iкомі, F., \& Winger, G. The reinforcing properties of ethanol. In M. K. Roach, W. M. McIssac, \& P. J. Creaven (Eds.), Biological aspects of alcohol. Austin: University of Texas Press, 1971.

(Manuscript received May 15, 1981; revision accepted for publication September 29, 1981.) 